\title{
Multilinguales
}

\section{Étude stylistique de la référenciation dans Le Chant du monde de J. Giono}

Stylistic Study of Referential Meaning in J. Giono's Le Chant du Monde (The Song of the World)

\section{Youssef El Yaacoubi}

\section{(2) OpenEdition}

\section{Journals}

Édition électronique

URL : http://journals.openedition.org/multilinguales/1336

DOI : $10.4000 /$ multilinguales. 1336

ISSN : 2335-1853

\section{Éditeur}

Université Abderrahmane Mira - Bejaia

\section{Édition imprimée}

Date de publication : 1 décembre 2014

Pagination : 169-189

ISSN : 2335-1535

\section{Référence électronique}

Youssef El Yaacoubi, «Étude stylistique de la référenciation dans Le Chant du monde de J. Giono », Multilinguales [En ligne], 4 | 2014, mis en ligne le 01 décembre 2014, consulté le 17 septembre 2019. URL : http://journals.openedition.org/multilinguales/1336; DOI : 10.4000/multilinguales. 1336

Ce document a été généré automatiquement le 17 septembre 2019

\section{(c) (†) $\odot$}

Multilinguales est mise à disposition selon les termes de la Licence Creative Commons Attribution -

Pas d'Utilisation Commerciale - Pas de Modification 4.0 International 


\title{
Étude stylistique de la référenciation dans Le Chant du monde de J. Giono
}

\author{
Stylistic Study of Referential Meaning in J. Giono's Le Chant du Monde (The \\ Song of the World)
}

Youssef El Yaacoubi

1 Dans son ouvrage Le Récit poétique (1978: 7), Jean-Yves Tadié étudie la prose poétique dans des œuvres où la poésie se mêle fortement à la prose romanesque et qu'il classe dans la catégorie " récit poétique »:

Le récit poétique en prose est la forme du récit qui emprunte au poème ses moyens d'action

et ses effets, si bien que son analyse doit tenir compte à la fois des techniques de description

du roman et de celles du poème: le récit poétique est un phénomène de transition entre le

roman et le poème.

Mais s'agissant de la prose poétique du roman, Dominique Combe, dans Poésie et récit, une rhétorique des genres (1989), affirme que cette prose poétique est le signe d'un retour du récit réconcilié avec la poésie, après leur rupture initiée, selon lui, par Stéphane Mallarmé notamment. Donc, pour lui, la poésie du récit n'est pas une simple transition :

C'est donc parce que la conception du roman s'est résolument écartée des canons « réalistes "

du siècle dernier que la poésie peut lui être assimilée : en réalité, c'est bien le roman qui a été "poétisé », conformé aux règles d'une poésie "pure» non référentielle, c'est-à-dire «lyrique ». La forme contemporaine de la poésie «pure " n'est autre que le Nouveau Roman. Rien de plus naturel que, alors que le roman se maintient comme forme dominante en raison de facteurs socio-économiques, la poésie se confond avec lui : en réalité elle se dissimule sous le pseudonyme trompeur de "roman». Rien de commun, alors, avec ce qu'on appelle quelquefois le "roman poétique" qui seul mérite la qualification de genre esthétique. (pp. 115-116)

De là, la nécessité de ne pas lire le roman et la poésie comme deux entités séparées dont il faudrait déchiffrer les relations interactionnelles qui les associeraient.

3 C'est dans cet état d'esprit que nous aborderons la problématique du «roman poétique " à travers l'exemple de Le Chant $d u$ monde $^{1}$ de Jean Giono, car l'auteur 
parvient, par le truchement de la description, prise au sens strict de représentation mimétique du réel, à représenter le monde et à l'investir d'un pouvoir poétique, voire mythique.

\section{Sublimation du paysage : zoomorphisme et anthropomorphisme}

4 Le paysage littéraire est une figure culturelle construite. Il constitue un iconotexte qui se définit par ses propres lois de fonctionnement et de représentation. Il est échafaudé selon le processus complexe de la description. Il est conçu comme une construction qui doit de nommer, évoquer et donner à voir. Il suppose donc des référents culturels d'autant la description est une construction qui s'adresse moins à l'intellect qu'à l'imagination et à la sensibilité du lecteur. Ce dernier ne cherchera pas à la comprendre mais plutôt à la visualiser car elle est bâtie selon le principe de l'iconicité qu'Yves Bonnefoy (1983:33) définit d'une manière lyrique :

J'appellerai image cette impression de la réalité enfin pleinement incarnée qui nous vient, paradoxalement, de mots détournés de l'incarnation. [...] Images, l'éclat qui manque à grisaille des jours, mais que permet le langage quand le recourbe sur soi, quand le pétrit comme un sein natal, la soif constante du rêve (...).

5 Ainsi, pour « donner cette impression de réalité », l'image du paysage, dans Le Chant du monde de Jean Giono, use pourtant d'artifices langagiers, « détournés de l'incarnation » et de la représentation mimétique du réel, comme nous tenterons de le démontrer.

L'intérêt de la lecture du paysage de Giono ne réside donc pas seulement dans le déchiffrement des éléments qui le constituent (les pentes, les couvertures végétales, les maisons du village, etc.), et de leur place dans le temps et l'espace, mais dans leur référenciation ${ }^{2}$, autrement dit dans la compréhension des modalités de leur agencement et de leurs symboliques.

Toutefois, interrogeons-nous d'abord sur sa définition. Augustin Berque, cité par AnneMarie Boisvert (2006) l'un des critiques de l'art paysager, détermine, dans son livre Les Raisons du paysage. De la Chine antique aux environnements de synthèse (1995), quatre critères qui définissent le paysage au sens propre :

1) Des représentations linguistiques, c'est-à-dire un ou deux mots pour dire " paysage" ;

2) des représentations littéraires, orales ou écrites, chantant ou décrivant les beautés du

paysage:

3) des représentations picturales, ayant pour thème le paysage ;

4) des représentations jardinières, traduisant une appropriation esthétique de la nature (il

ne s'agit donc point de jardins de subsistance).

D'après Anne-Marie Boisvert, on doit cette présentation des critères à Alain Roger dans son Court traité du paysage (1997: 48). Quant à elle, elle cite (2006) Augustin Berque (1995 : 34-35) qui constate que

Tel ou tel des trois derniers critères peut se retrouver dans de nombreuses sociétés; mais

c'est seulement dans les sociétés proprement paysagères, qui sont aussi les seules à présenter

le premier, que l'on trouve réuni l'ensemble des quatre critères.

De ces considérations, il est donné à comprendre que le paysage est un topos culturel par excellence. Il est à la fois textuel et référentiel. En texte, le paysage propose son propre discours. C'est ainsi que Giono, dans son récit, mêle intimement le narratif et le descriptif. En effet, le descriptif se greffe sur le narratif tantôt en le suspendant, tantôt en le ralentissant et tantôt en lui assurant continuité et fluidité. C'est le cas de la description du fleuve du point de vue du narrateur omniscient : 
La nuit, le fleuve roulait à coups d'épaules à travers la forêt, Antonio s'avança jusqu'à la pointe de l'île. D'un côté, l'eau profonde, souple comme du poil de chat; de l'autre côté les hennissements du gué. Antonio toucha le chêne. Il écouta dans ses mains les tremblements de l'arbre. C'était un vieux chêne plus gros qu'un homme de la montagne. [...] Le fleuve qui sortait des gorges naissait dans un éboulis de montagne. C'était la haute vallée noire d'arbres noirs, d'herbe noire et de mousse pleine de pluie. Elle était creusée en forme de main, les cinq doigts apportent toute l'eau de cinq ravinements profonds dans une large paume d'argile et de rochers où le fleuve s'élançait comme un cheval en pataugeant avec ses grands pieds pleins d'écume. (p. 7)

La description use, ici, d'artifices de la rhétorique pour donner une dimension isotope à tous les éléments. A la faveur de métaphores et de comparaisons, plusieurs isotopies concourent à animer une composition en noir (« nuit», "vallée noire ", " arbres noirs », « herbe noire ») :

- l'isotopie de l'énergie qui associe les sèmes de puissance/force (" coups d'épaule », "vieux chêne ", «montagne ", « gros", "grand», « rocher », ...), aux sèmes du mouvement ( « roulait », « s'avança », « toucha », « sortait des gorges », « s'élançait », « pataugeait »),

- l'isotopie zoomorphe qui associe certains éléments de la nature à des animaux ( « eau...comme poil de chat », « hennissement du gué », « fleuve...comme un cheval »),

- l'isotopie anthropomorphe dont l'effet est la fusion du personnage dans le paysage («il écouta...les tremblements de l'arbre...plus gros qu'un homme de la montagne ", «il (Antonio) écouta dans ses mains »/ « elle (la haute vallée) était creusée comme une main », " large paume d'argile»).

Ainsi, nous pouvons dire que ces trois isotopies principales partagent le sème de / mouvement/, qui, combiné à la puissance assure la cohérence de la description.

Cette description dépasse le cadre d'une quelconque reproduction mimétique, à la faveur, en premier lieu, du procédé de la comparaison, définie ainsi par Jean Kokelberg (1993: 82) :

Mode d'expression qui consiste à établir - par une CHARNIERE GRAMMATICALE ad hoc - un rapprochement fondé sur la perception d'une analogie entre deux réalités ou deux idées. Le rapprochement ainsi opéré trouve sa justification dans le désir de voir - ou de représenter avec plus de force - l'objet, l'idée, l'action ou la situation que l'on cherche à évoquer.

Il précise que la contiguïté des deux réalités opérée par la comparaison «associe de manière intime et inattendue des mots ou des idées appartenant à des registres différents » (idem.).

A la faveur, notamment, de la charnière grammaticale "comme ", qui rapproche " eau » et "chat ", "fleuve » et " cheval », le narrateur donne " plus de «force " au contraste entre les deux comparés : la souplesse (féline) de l'eau profonde à « la pointe de l'île" s'oppose à la lourdeur maladroite et massive du fleuve qui foule l'eau " pataugeant avec ses grands pieds pleins d'écume", expression qui se laisse lire, par association, " pataugeant avec ses gros sabots pleins d'écume ».

6 Ainsi l'écriture de Giono donne forme à sa propre esthétique du paysage poétique. En effet, la métaphore de la vallée anthropomorphique, qui placée sous le régime nocturne, acquiert un aspect naturel et sauvage. La comparaison avec la main permet d'articuler, par le jeu des comparatifs, une véritable rhétorique humanisant la nature. De plus, la structure répétitive des lexèmes amplifie l'effet des comparatifs.

Cet anthropomorphisme est accentué par les registres et les codes de la description qui s'insinuent au niveau narratif. La description raconte l'histoire du fleuve qui accomplit un certain nombre d'actions (rouler, sortir des gorges, naître) à travers la forêt, la 
montagne, jusqu'à la vallée, sous l'œil complice du personnage. En effet, selon Adam et Petitjean (1992 : p. 41)

La description est prise en charge dans ce cas, par un acteur doué de la possibilité de voir, d'observer. Ce qui ne va pas sans rejaillir sur le choix des qualifications du voyeur (peintre, badaud, espion (...) et sur les motivations (curieux, intrigué, nouveau venu (...). A quoi il faut ajouter que le personnage en question doit être placé dans un milieu ambiant favorisant son penchant à l'observation (lieu élevé, ouverture, milieu transparent (...) et accomplir une action- prétexte caractéristique (...).

A cette "possibilité de voir, d'observer ", s'ajoute, dans notre séquence, la possibilité d'écouter («il écouta »), la possibilité de toucher («il toucha). Ces deux aptitudes sensorielles, contrairement à leurs référents dans le monde tangible, n'en font qu'une dans le texte (puisqu'Antonio écoute avec ses mains qui touchent le chêne), qui produit de ce fait une sensation sans référence, inédite. Cette écoute l'est d'autant plus qu'elle s'exerce sur un « chêne », symbole de solidité massive inébranlable dans l'imaginaire collectif, par des « mains » à même de sentir, voire de provoquer, ses «tremblements ». De plus, le récit se passe en un temps (la nuit) et en un espace (arbres, herbe, vallée) marqués chromatiquement. En effet, ce récit est combiné aux effets visuels de la noirceur intensifiés par la répétition de l'adjectif «noir» qui s'étend sur tout le paysage.

Nous n'avons pas perdu de vue la recommandation de Jean- Michel Adam dans son ouvrage Langue et littérature, analyses pragmatiques et textuelles (1991:131):

Après cette description de la cohésion et de la progression [...], il faut se demander si les isotopies de base qu'une première lecture dégage immédiatement (anthropos et cosmos) sont les seules identifiables.

Nous avons donc continué à interroger l'œuvre de Giono et nous pouvons affirmer que les isotopies que nous venons d'identifier se retrouvent au niveau de toute l'œuvre et qu'elles ne sont pas les seules à sublimer le paysage en lui assurant une cohérence constante.

\section{Sublimation du paysage : I'hyperbole de la lumière}

Un autre procédé stylistique qui a trait à la mythification du paysage dans le texte de Giono est l'hyperbole dont voici un exemple :

À peine sorti de l'horizon, le soleil écrasé par un azur terrible ruisselait de tous côtés sur la neige gelée; le plus maigre buisson éclatait en cour de flamme. Dans les forêts métalliques et solides le vent ne pouvait pas remuer un seul rameau (...). Des poussières pleines de lumières couraient sur le pays. (...) Les bêtes s'arrêtaient en plein soleil avec leurs poils tout salés de neige gelée, dure comme une poussière de granit... Le jour ne venait plus du soleil seul, d'un coin $\mathrm{du}$ ciel, avec chaque chose portant son ombre, mais la lumière bondissait de tous les éclats de la neige et de la glace dans toutes les directions et les ombres étaient maigres et malades, toutes piquetées de points d'or. On aurait dit que la terre avait englouti le soleil et que c'était elle, maintenant, la faiseuse de lumière. On ne pouvait pas la regarder. Elle frappait les yeux: on les fermait, on la regardait de coin pour chercher son chemin et c'est à peine si on pouvait la regarder assez pour trouver la direction: tout de suite le bord des paupières se mettait à brûler et, si on s'essuyait l'œil, on se trouvait des cils morts dans les doigts (p. 138)

Au niveau des intensifs et des comparatifs, notons :

- d'abord, la radicalité et la violence des signifiés narratifs : "écrasé », " éclatait ", " ne pouvait pas", «s'arrêtaient », «englouti", «fermait», « ne pouvait pas », "courir sur le pays », « frappait », « brûlait », « trouvait morts »; 
- ensuite, la plénitude spatio-temporelle qui encadre cette séquence : «azur terrible», «tout côté », " pleines de lumière ", " plein soleil », " tous les éclats ", «toutes les directions », «toutes piquetées»;

- enfin, l'extrême dureté de la consistance des éléments qui composent le paysage : "neige gelée ", "forêts métalliques et solides", "neige gelée dure comme une poussière de granit ", « glace », " points d'or » "; Le sème de la dureté est associé à celui de l'immobilisme renforcé par le contraste avec l'extrême agilité de la lumière (« couraient », « bondissait »).

Ce processus d'emphatisation hyperbolique du paysage est dominé par l'irradiation de la lumière intensifiée par sa réflexion sur la glace. Son intensité étend son emprise sur toute la surface de la terre et confère au paysage une dimension épique par la radicalité antagoniste de ses composants.

En effet, le paysage, immobile, contraste avec l'agilité de la lumière: "les forêts métalliques et solides » où «le vent ne pouvait remuer aucun rameau ", «les bêtes " recouvertes de " poussière de granit » semblent subir les assauts d' « un azur terrible » qui « écrase » le " soleil qui ruisselle ", et dont « la lumière bondissait de tous les éclats de la neige et de la glace ». Le mouvement anime le récit et lui donne une vie intense.

La lumière minérale (neige - glace), dans son intensité, a évincé la lumière sidérale (soleil), a minéralisé le paysage et les animaux et menace les humains qu'elle oblige à vivre dans l'obscurité. Ils ne peuvent, en effet, regarder la lumière et sont contraints de "se bander" pour pouvoir supporter son intensité mortelle. L'importance du regard associée à la pétrification dans cet espace glacial sous un azur terrible confine cette lumière au rang de mythe : celui de Méduse qui pétrifie les mortels qui lèvent les yeux sur elle, comme la lumière le fait pour les « cils» métonymiques des hommes (« on ») qui osent la fixer.

Cette lumière de « la terre ", plus forte que le soleil (« écrasé », « englouti »), ce paysage tout entier, règne sur un paysage métaphoriquement « médusé », élevant l'ensemble au rang de mythe.

Ainsi, l'hyperbolisation jusqu'à la mythification anime le paysage en modulant sa description selon des signifiés narratifs mythiques, comme dans l'exemple de Méduse dans la séquence ci-dessus.

Ce procédé d'hyperbolisation s'étend non seulement à d'autres descriptions de paysages de l'œuvre de Giono mais aussi, et de façon plus prégnante que dans cette séquence, aux hommes. Citons, à titre d'exemple, la description du bouvier en hiver :

Le bouvier avait une toque en poils d'ours, ses deux gros foulards noués l'un sur l'autre, sa veste de cuir, des moufles en peau de mouton et des cuissards de loutre ficelés le long de ses jambes depuis la cheville jusqu'à la hanche. (p.144)

Physiquement, il est de complexion animale: les sèmes constitutifs des métonymies relevant de l'isotopie zoomorphe ("poils d'ours", "veste de cuir", "peau de mouton", "cuissards de loutre ") achèvent d'assimiler l'homme, de la tête (" toque ») aux pieds ("chevilles »), à un animal à la fois terrestre et aquatique, carnivore et herbivore, sauvage et domestique, massif et souple, etc. Cet animal n'existe bien entendu que par la magie paragrammatique qui condense, dans les signifiants de la première proposition, les lexèmes d'« ours », de "peau » et d'« outre", dominants de par leur proportion physique : la peau en tant que métonymie à la fois d'ours, de veste, de mouton et de loutre, et les cuissards qui recouvrent les jambes.

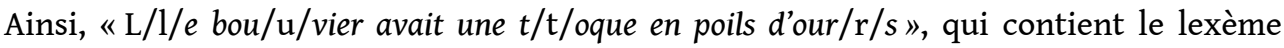
" ours ", le paragramme de « loutre» $(/ \mathrm{l} / \mathrm{u} / \mathrm{t} / \mathrm{r} /)$, et de « peau » $(-$ « p/p/o/o/ils»), fait $\mathrm{du}$ « bouvier » un animal, à la fois ours et loutre, de par les peaux dont il est recouvert. 
Cet animal fictif auquel est associé le bouvier intensifie son caractère bestial en rapport avec son rôle dans le récit.

Cette hyperbolisation des hommes et du paysage peut, à certains moments clés du récit, être portée au niveau supérieur de l'allégorie, voire au niveau suprême de la parabole, comme la parabole biblique de la Genèse que nous étudierons dans le point suivant.

\section{Sublimation du paysage : la parabole de la genèse}

Les valeurs paysagères dans Le Chant $d u$ monde sont de l'ordre pictural et photographique surtout lorsqu'il s'agit du paysage figé dans le silence par la neige et la glace de l'hiver. En effet, de nombreuses descriptions paysagères sont travaillées par le modèle de la photographie. Giono utilise le phénomène optique du focus pour organiser sa description en plusieurs plans et points de vue, avec des effets d'agrandissement des premiers plans et de réduction des arrières plans. En voici un exemple :

Il y avait un grand silence. Les branches encore sans feuilles étincelaient de mille petites

flammes d'argent et, sous chaque flamme, dans la goutte d'eau brillante, les bourgeons neufs

se gonflaient. (p. 259)

La focalisation est centrée sur "les branches ", au premier plan, plus imposantes (en nombre, en quantité, en qualité et en volume: mille petites flammes d'argent, puis se resserre, au second plan, sur le niveau inférieur de la goutte d'eau brillante et enfin sur ce qu'elle abrite, les bourgeons. Il s'agit d'un paysage hivernal hiérarchiquement structuré selon un champ vertical descendant, qui baigne dans le silence total, évoquant la photographie en noir et blanc et en surbrillance.

Cependant, le récit reprend tout de même ses droits à la fin du tableau: le verbe gonflaient, par son aspect inchoatif, inscrit ce paysage photographique dans le temps annonciateur d'une nouvelle saison. L'écriture de ce changement de saison est surdéterminée (sur des dizaines de pages) par la parabole de l'origine de l'humanité telle que racontée dans le Livre de la Genèse (Ancien Testament). A cette parabole sera travaillée par une métaphore parabolique proprement textuelle, comme nous le verrons un peu plus bas.

Notre analyse des deux paraboles s'appuiera sur une démarche componentielle qui donne la priorité (mais non l'exclusivité) aux contenus des lexèmes. Les lexèmes seront étudiés dans un même environnement textuel, pour cerner leurs contaminations mutuelles et mettre en valeur leur transformation sémantique, induisant leur transmutation.

Disons donc, à ce niveau, que le printemps est décrit comme une renaissance du pays après le déluge provoqué par la pluie et la fonte des neiges (pp. 259-262), un événement cyclique que Giono va transfigurer en convoquant l'intertexte de la Genèse de façon quasi explicite.

En effet, le texte de Giono évoque à maintes reprises « la falaise de l'arche »/ «grand rocher tapissé de lierre et de clématite», refuge des oiseaux au moment où "des torrents musclés (...), plus haut que les sapins, (...), emportaient des lambeaux de forêts » (p.261), et qui rappelle l'arche biblique soulevée par la crue des eaux et déposée sur la montagne ${ }^{3}$. Cette association est renforcée par un certain nombre d'éléments contigus.

La description du paysage n'est pas impersonnelle, mais modalisée, en amont, par le sentiment dysphorique inspiré par le déluge : 
Il y avait de quoi être grave et inquiet. Les eaux n'avaient fait que monter tout le jour. (...) Sur le visage des hommes les femmes regardaient peureusement cette gravité et ce souci. (p. 228)

Cette modalisation confère à la crue des eaux le sème de /menace/ (ou /danger/) justifié par son ampleur et sa violence. Comme dans la Genèse, il pleut: «De grandes pluies grises traversaient le ciel. Tout disparaissait : montagnes et forêts » (p.259) ; cette pluie et la fonte des neiges entraînent une crue dont le niveau couvre les montagnes, comme dans la Genèse.

La "pluie » s'accompagne de la «bise » qui ajoute au "vent » de la Genèse, les sèmes contextuels de /froid/. Mais, contrairement au «vent» de la Genèse qui chasse la pluie et contribue ainsi à l'apaisement de l'univers, la «bise noire » du Chant du monde est un mauvais présage dont la présence est prégnante. Lexicalisée par ses nombreuses répétitions contigües, elle rythme chronologiquement tout le récit du déluge en constituants ses charnières : 1- « La bise noire était arrivée de l'est » (p. 259), 2- « La bise se relevait » (p. 260), 3- « la bise retombait de tout son poids» (p. 260), 4-, « La bise s'arrêta. (...). Les derniers soubresauts de la bise secouaient quelques tringles de grêles » (pp. 260-261).

Certes, la fin de cette « bise noire » signe le renouveau, comme le vent de la Genèse :

Le soleil reprenait de jour en jour sa couleur naturelle. (...) Les bêtes de poils, les bêtes de plumes, les bêtes de peau rase, les bêtes froides, les bêtes chaudes, les perceurs de terre, d'écorces, de roches, les nageuses, les coureuses, les voiliers: tout commençait à nager, à courir, à voler avec des souvenirs d'anciens gestes. (p.261)

Mais, au contraire du vent de la Genèse qui chasse les dernières séquelles du déluge, la « bise noire » signe aussi le début du déluge :

Les glaciers fondaient. (...) la montagne couverte de cascades grondait comme un tambour. Il n'y avait plus de petits ruisseaux mais des torrents musclés aux reins terribles et qui portaient des glaçons et des rochers, bondissaient, luisants et tout fumants d'écume plus haut que les sapins, minaient leurs rives profondes, emportaient des lambeaux de forêts. Les eaux, les roches, les glaces, les ossements d'arbres se tordaient en grosses branches d'acier à travers le pays et se déversaient en mugissant dans l'immense fleuve. (p. 266)

L'expression "gronder comme un tambour", qui convoque celle, référentielle, de "résonner comme un tambour», ajoute au son caractéristique du comparant ( "tambour ») le sème de /menace/ au comparé (" montagne »). Les prémices d'hostilité se concrétisent dans « des torrents musclés [qui] minaient leurs rives profondes, emportaient des lambeaux de forêts", "les eaux, les roches, les glaces, les ossements d'arbres [qui] se déversaient en mugissant dans l'immense fleuve ", associés à la violence mortelle exercée sur le paysage (minaient, emportaient des lambeaux, ossements d'arbres).

Le renouveau dans Le Chant du monde est au prix de ces crues dévastatrices comme dans la Genèse, et comme dans la Genèse, ses prémices se manifestent sur la falaise de l'arche, matrice de l'univers, peuplée de tous les animaux avec une mention spécifique pour ceux de la Genèse : les oiseaux, les poissons, les corbeaux, etc. :

Depuis longtemps les houldres avaient quitté la falaise de l'arche pour aller crier le printemps partout. Mais les oiseaux ordinaires revenaient tous les soirs au grand rocher tapissé de lierre et de clématite. Il y avait des fauvettes, des mésanges de toutes les sortes, des rossignols, des verdiers, des carmines, des pies, des corbeaux, tous les habitants de la ronce ou de la forêt, mais rien que des mangeurs de viande. Pas des mangeurs de graines. Ils étaient gras et lourds à ne plus bien savoir ni voler ni marcher. Ils se cramponnaient dans les résilles de branches et de feuilles qui tapissaient le rocher et ils restaient là un moment à se reposer $d u$ vol de tout le jour sur le grand pays plein de chaleur et d'espérance. (pp. 261-262)

Aux nombreux éléments empruntés à la Genèse, dont nous n'avons repris qu'une infime partie, nous devons ajouter à celui, cardinal, de l' «arc-en-ciel», pour achever 
d'emporter la conviction : «La pluie nouvelle venait à travers les sapins, la bise retombait de tout son poids, les taches noires de la pluie et du soleil marchaient dans tout le pays sous une frondaison d'arcs-en -ciel » (p. 260).

A l'instar de l'arc-en-ciel, signe divin créé à la fin du déluge dans la Genèse pour sceller l'union sacrée, les «arcs-en-ciel » du Chant du monde apparaissent eux aussi au moment de la décrue mais dans une version terrestre («marchaient»), désacralisée par sa profusion (frondaison d'arcs-en-ciel), mais pour sceller également une union : celle du ciel et de la terre.

Nous avons jusqu'ici souligné, certes de façon sommaire tant les éléments intertextuels sont nombreux, ce que le passage de l'hiver au printemps devait à la parabole biblique. Mais celle-ci est insérée dans un autre processus métaphorique propre au texte de Giono, comme nous allons tenter de le démontrer en étudiant les figures dans un même environnement textuel.

En effet, dans Le Chant du monde, le passage de l'hiver au printemps se lit comme celui de la bauge à la falaise de l'arche, l'orage ayant permuté explicitement avec le sanglier, auquel il continue tout de même à se superposer, par association, dans l'expression " l'orage se dressait dans sa bauge » (p.259). Aux sèmes /fangeux/ et /boueux/, attachés au lexème bauge, dans l'imaginaire collectif, vont, par contamination sémantique, s'ajouter deux autres sèmes isotopes: /noirceur / et /piétinement/. Notons qu'il s'agit davantage d'une mise au jour de ces sèmes plutôt que d'une nouvelle acquisition. Ces sèmes déterminent, par association, un champ lexico-sémantique qui entraîne dans son sillage les moments du déluge : avant, pendant et après (l'après caractérisé par son opposition aux deux premiers moments).

Ainsi, " orage " fonctionne comme un archilexème dont les archisémèmes seront déterminés à partir de "l'orage se dressait dans sa bauge »" et en suivant sa contamination dans son environnement lexico-sémantique.

Le tableau ci-dessous nous aidera à expliciter notre propos :

\begin{tabular}{|l|l|l|l|}
\hline extraits (Le Chant du monde) & sèmes & lexèmes & $\begin{array}{l}\text { pages } \\
\text { contigües }\end{array}$ \\
\hline L'orage se dressait dans sa bauge & $\begin{array}{l}\text { /boueux/ } \\
\text { fangeux/ }\end{array}$ & orage & 260 \\
\hline Il piétinait les villages et les champs & /piétinement/ & orage & 260 \\
\hline piétinement léger des oiseaux & /piétinement/ & oiseaux & 260 \\
\hline le piétinement de la pluie passée descendait vers les fonds & /piétinement/ & pluie & 260 \\
\hline taches noires de la pluie & /noir/ & pluie & 260 \\
\hline $\begin{array}{l}\text { La pluie pendait sous la bise comme les longs poils sous le ventre } \\
\text { des boucs }\end{array}$ & $\begin{array}{l}\text { /puanteur/ } \\
\text { /lubricité/ }\end{array}$ & boucs & $259-260$ \\
\hline $\begin{array}{l}\text { /souillure/ } \\
\text { piétinement léger des oiseaux }\end{array}$ & $\begin{array}{l}\text { /noir/ } \\
\text { /piétinement/ }\end{array}$ & Terre & 260 \\
\hline bise noire & /noir/ & Bise & 259 \\
\hline
\end{tabular}




\begin{tabular}{|l|l|l|l|}
\hline boue noire & /boueux/noir/ & Boue & 270 \\
\hline nuage sombre & /noir/ & Nuage & 259 \\
\hline nuage de boue & /boueux/ & Nuage & 270 \\
\hline ciel boueux & /boueux/ & Ciel & $177-211$ \\
\hline
\end{tabular}

L'orage (boueux et fangeux) est sémantiquement associé au «ciel boueux» qui revient comme un leitmotiv en amont de la séquence du déluge. Le "ciel boueux», devenu fangeux par son association à "l'orage », convoque par association «nuage de boue ", " boue noire " pour acquérir le sème /noir/, confirmé par « il (le ciel) n'était pas là-haut à sa place comme d'habitude mais contre terre » (p. 237).

"Ciel » est associé à "l'orage" "[qui] piétine les champs et les villages", et aux "oiseaux [qui] piétinent une terre noire et boueuse»; d'où, par contamination, un [ciel boueux, noir, qui piétine].

«Orage» et «ciel» sont associés à la "pluie noire» qui «piétine en descendant vers les fonds»; "pluie noire» et "terre noire», associés, convoquent dans leur sillage "bise noire ». De par cette association, "L'orage» est sémantiquement contaminé et se lit: [l'orage boueux, fangeux, noir qui piétine dans sa bauge].

La "bise noire», associé "la pluie noire qui piétine ", l'est également avec les autres éléments qui "piétinent " ("l'orage» et oiseaux»), et à tous les éléments contigus du paysage qui sont de la même couleur (nuage, pluie, terre, boue). Ainsi «bise noire », est contaminé par les sèmes /piétinement/ et /boueux/. «La bise noire » se lit alors [la bise noire, boueuse, fangeuse, piétine dans sa bauge].

Ainsi, avant le déluge, et par contamination sémantique, le paysage est constitué de l'orage, du ciel, de la pluie, des oiseaux, de la terre. Ils sont boueux, fangeux, noirs, et piétinent dans leur « bauge »fangeuse, boueuse et noire.

En outre, dans le contexte des faisceaux sémantiques articulés autour de la "bauge », le rapprochement de "pluie» avec "bouc» («La pluie pendait sous la bise comme les longs poils sous le ventre des boucs ») confère au paysage, entre ciel et terre, les sèmes du sémème /bouc/, tels que /lubricité/5/impureté//puanteur/péché/ ${ }^{6}$.

La convocation de ces sèmes est également favorisée par la proximité phrastique, immédiatement en amont, de "De grandes pluies grises traversaient le ciel», et de la contiguïté de "pendait sous le ventre», inscrivant alors la "bauge » de "l'orage» dans le champ de la religion en la chargeant des sèmes contigus de /lubricité/ et de / souillure/. De ce fait, le paysage entier est une [bauge fangeuse, boueuse, noire, impure, fétide] qui va subir le déluge comme une purification par l'eau. C'est ce qui est donné à lire dans le renouvellement des composants du paysage :

- la pluie : «la pluie nouvelle » se substitue à « la pluie passée », antédiluvienne : «le piétinement de la pluie passée descendait vers les fonds. La pluie nouvelle venait à travers les sapins» p. 260) ;

- le soleil : « Le soleil reprenait de jour en jour sa couleur naturelle » (p. 261);

- le ciel : «le sable fin du ciel dégagé » (p. 261);

- «la frondaison d'arcs-en-ciel » de la «pluie » et du «soleil » remplacent les «longs poils sous le ventre des boucs ».

Le printemps peut alors naître à l'endroit même de la bauge antédiluvienne, que le déluge a emportée : «c'était seulement le printemps qui sortait de la terre 》(p. 259). Le récit de l'arrivée du printemps, ainsi banalisée par la locution restrictive (ne...que), acquiert 
de l'intensité par euphémisme, eu égard à la charge symbolique de son lieu de naissance.

Nous pouvons donc en déduire que, grâce à tous ces faisceaux lexico-sémantiques, la parabole de la Genèse est tissée de la métaphore de « la bauge » qui est, de ce fait, hissée au rang de parabole, en dépit de la trivialité de son designatum, dans son association au déluge et par contamination. L'arche et la bauge sont les deux pôles contraires d'un même continuum. L'originalité du Chant du monde tient au fait qu'il ait investi le moment de l'entre-deux, après l'hiver mais avant le printemps, un temps interstitiel où la fiction installe la bauge pour combler le vide de l'allégorie.

11 Au terme de ce parcours, nous pensons avoir montré par quels procédés stylistiques de référenciation et pour quels effets Le Chant du monde décrit le paysage. Nous pouvons conclure que Giono ne concurrence pas la réalité sur son terrain, mais il la transfigure pour en produire une nouvelle qui n'existe que dans le texte.

\section{BIBLIOGRAPHIE}

A travers l'étude stylistique des figures (métaphore, comparaison, ellipse, hyperbole, parabole, etc.), dans leurs modalités de présence en texte, à travers leurs faisceaux lexico-sémantiques, leurs processus de contamination, nous avons tenté de mettre au jour la manière dont Jean Giono, dans Le Chant du monde, parvenait non seulement à dramatiser son récit mais à lui conférer une dimension épique. Il tisse le narratif, le descriptif, le référentiel et le mythique de façon à la fois originale et en recourant aux lieux communs de l'imaginaire collectif.

ADAM Jean-Michel, Langue et littérature, analyses pragmatiques et textuelles, Paris, 1991.

ADAM Jean-Michel, PETITJEAN André, Le texte descriptif, Paris, Nathan, coll. « Littérature », 1992.

AQUIN (D') Thomas, «Question 102 : les raisons d'être des préceptes cérémoniels ", dans Somme théologique, Prima Secundae, 1269-1270. Consulté sur le site : < http://www.santorosario.net/ somme/primasecundae/102.htm >

BERQUE Augustin, Les Raisons du paysage. De la Chine antique aux environnements de synthèse, 1995.

BOISVERT Anne-Marie, «CARLO ZANNI OU LE PAYSAGE DU NOVO MILLENNIO », mis en ligne en octobre 2006. Disponible sur le site : < http://www.zanni.org/html/txtcritics/boisvert2.htm >

BONNEFOY Yves, Récits en rêve, Paris, Gallimard, 1977.

BONNEFOY Yves, La présence de l'image, Mercure de France, 1983. COMBE Dominique, Poésie et récit, une rhétorique des genres, Paris, José Corti, 1989.

CRESSOT Marcel, JAMES Laurence, Le Style et ses techniques, Paris, PUF, 1991.

Dictionnaire Larousse étymologique et historique, Librairie Larousse, 1971.

KOKELBERG Jean, Les Techniques du style, Paris, Nathan, coll. « Littérature », 1993.

MILLY Jean, Poétique des textes, Paris, Nathan, 1992. 
PEYTARD Jean, « Iconicité et référenciation (aux limites de l'écriture) », in Semen [En ligne], 4 | 1989, mis en ligne le 11 novembre 2008. Consultable sur le site $:<$ http://semen.revues.org/6823 > PRIEUR Jean, Les symboles universels, Fernand Lamore, 1989.

ROGER Alain, Court traité du paysage, Bibliothèque des sciences humaines, NRF, Gallimard, 1997

TADIE Jean -Yves, Le récit poétique, PUF, 1978.

CORPUS : GIONO Jean, Le Chant du monde, Paris, Gallimard, 1997.

\section{NOTES}

1. Dans la présente étude, nous nous référons à l'ouvrage des éditions Gallimard, 1997.

2. Nous reprenons la définition de Jean Peytard: "Supposons, écrit-il, qu'un même événement sportif soit l'occasion de trois reportages, chacun utilisant un canal différent: l'un la radio, l'autre la télévision, le troisième la presse imprimée. Nous dirons que cet événement constitue le référent ; que l'outillage discursif utilisé caractérise la référenciation; que le produit discursif présenté à un public est le référé. Trois termes : référent, référenciation, référé, que nous utiliserons avec le sens que nous venons de leur donner » (mis en ligne en 2008).

3. Des exégètes ont identifié cette montagne au Mont Ararat.

4. Bauge : " de bauche, gîte fangeux du sanglier ", " terre boueuse », Dictionnaire Larousse étymologique et historique, Librairie Larousse, 1971, p. 78.

5. "Le bouc est définitivement rangé parmi les animaux diaboliques. Il est définitivement symbole de la lubricité et du mensonge » (Prieur, 1989 : 29).

6. «On sait que le bouc est une bête fétide et que les vêtements tissés de son poil étaient irritants pour la peau : cela signifiait la puanteur, l'impureté et l'aiguillon du péché » (Thomas d'Aquin, 1269-1270).

\section{RÉSUMÉS}

Notre étude porte sur la référenciation dans l'œuvre de Jean Giono, Le Chant du monde parce qu'elle relève de divers modes de représentation. Nous tentons de démontrer que le référent se transmue par la magie du style et de ses techniques en un référé qui intègre l'intertexte mythique. En effet, les relations interactionnelles investissent la représentation par le biais de procédés stylistiques, notamment la comparaison et la métaphore, pour présenter une "réalité » autre, un monde doté d'un pouvoir poétique, voire mythique. En cela, le paysage, dans Le Chant $d u$ monde de Jean Giono, en tant que "référé ", se lit comme une image harmonieuse du monde grâce à la description/création des lieux marqués par la cohabitation parfaite des êtres et des choses.

Our paper, aided by work in linguistics and stylistics, focuses on referential meaning and telescoping of a reality other than what is stated openly in the text. To do this, we chose the work of Jean Giono, a great creator of images and spaces, Le Chant du Monde, where the narrative incorporates the poetic dimension through different modes of representation, whether literary, poetic musical or pictorial. Furthermore, representation is transmuted by the magic of style and 
techniques in a reality that concerns the mythical intertext. In this, the landscape in Le Chant $d u$ Monde by Jean Giono, the description of the places in his bill incorporates the co-presence of the human and the animal in perfect harmony relating to myth and perceptions.

\section{INDEX}

Keywords : referential meaning, description, poetic, stylistic, intertext, Le Chant du monde, Giono (Jean)

Mots-clés : référenciation, description, poétique, stylistique, intertexte, Le Chant du monde, Giono (Jean)

\section{AUTEUR}

YOUSSEF EL YAACOUBI

Université Sidi Mohamed Ben Abdellah Fès - Maroc 